\title{
Localization in an imaginary vector potential
}

\author{
P.G.Silvestrov \\ Budker Institute of Nuclear Physics, 630090 Novosibirsk, Russia
}

\begin{abstract}
Eigenfunctions of $1 d$ disordered Hamiltonian with constant imaginary vector potential are investigated. Even within the domain of complex eigenvalues the wave functions are shown to be strongly localized. However, this localization is of a very unusual kind. The logarithm of the wave function at different coordinates $x$ fluctuates strongly (just like the position of Brownian particle fluctuates in time). After approaching its maximal value the logarithm decreases like the square root of the distance $\overline{\left(\ln \left|\psi_{\max } / \psi\right|\right)^{2}} \sim\left|x-x_{0}\right|$. The extension of the model to the quasi- $1 d$ case is also considered.
\end{abstract}

PACS numbers: 72.15.Rn, 05.40.+j, 74.60.Ge

The non-Hermitean disordered Hamiltonians with imaginary vector potential were introduced in Ref. [1] for the description of vortex depinning from columnar defects by a transverse magnetic field in superconductors (later the analogous equations were used in the context of investigation of some problems of biological growth [2]). The features of this unusual Hamiltonians have attracted the immediate and wide interest [3 10]. From the theoretical point of view the most exciting was the prediction of the existence of a mobility edge in $1 d$ in such systems. However, the main attention in the existing literature was paid to the solution of eigenvalue problem in the imaginary potential. The spectrum of this non-Hermitean Hamiltonian was shown to consist of two segments of real eigenvalues and two arcs of imaginary eigenvalues (see insert on the Fig. 1). The domain of real energy was calculated in the initial paper Ref. [1]. The analytic formula relating the real and imaginary part of complex eigenvalues for $1 d$ model with weak disorder was found in Ref. [5] (some other analytic approaches and approximations were considered in Refs. [1, 1,8 ). While all eigenstates with real energy turn out to be naturally localized, the complex eigenvalues are usually associated with the extended states. Nevertheless, in this note we show that even after the transition to complex spectrum the wave functions remain sufficiently localized in $1 d$ imaginary vector potential. This localization, however, is of a very unusual kind. The logarithm of the wave function changes randomly with the increase of $x$. The maximum of the wave function corresponds to the maximal positive deviation of this random path from the initial position. After approaching maximum the logarithm of the wave function decreases also randomly being $\overline{\left(\ln \left|\psi_{\max }\right|-\ln |\psi(x)|\right)^{2}} \sim\left|x-x_{\max }\right|$. We will call the localization of the eigenvectors of this type the Stochastic Localization.

The Schrödinger equation for a particle in one channel disordered ring with constant imaginary vector potential has the form

$$
H \psi=-\left(\frac{\partial}{\partial x}-h\right)^{2} \psi+V(x) \psi=E \psi
$$

The boundary conditions are $\psi(x+L)=\psi(x)$ (the closed ring). For wire with open ends the vector potential may be washed out by the gauge transformation $\psi(x) \rightarrow \exp (h x) \psi(x)$. Our results do not depend on the concrete choice of the disordered potential $V(x)$ (it may be white noise or something else). Also for analytic estimates we consider the case of weak disorder. This means that both the wave length and $h^{-1}$ are small compared to the mean free path.

We will consider only the states with large complex energy. Eigenvectors with small real energy may be obtained by gauge transformation from the usual localized states with $h=0$. The most clear difference of the Hamiltonian Eq. (11) from the usual $1 d$ disordered case is that at $h$ large enough the left going and right going waves are no more divergent. The corresponding energies for the momentum $\pm k$ are $E_{ \pm}=k^{2}-h^{2} \pm 2 i k h$. As a result one may look for the solution of Eq. (1) of the form (analog of the WKB method in Hermitean Quantum Mechanics [13])

$$
\psi=\exp (i \sigma)
$$

The small corrections due to back scattering may be estimated by the method which we use below for the investigation of multichannel case. The derivative expansion for $\sigma$ now gives

$$
\begin{gathered}
\sigma=\sigma_{0}+\sigma_{1}+\ldots, \\
\sigma_{0}=\int_{0}^{x}(\sqrt{E-V}-i h) d x^{\prime} ; \sigma_{1}=\frac{i}{4} \ln (E-V) \ldots
\end{gathered}
$$

The weak disorder may mix only the plain-wave states with momentum $k^{\prime}$ close to $k$. Therefore, only slow garmonics of the potential $V_{k k^{\prime}}$ came into play, which justifies the use of WKB approximation [14]. For our purposes it will be enough to consider only the first term $\sigma_{0}$ of the expansion Eq. (3). The quantization condition now takes the form $\sigma(L)-\sigma(0)=2 \pi n$, which in the first order in $V$ leads to the energy $E=(k+i h)^{2}+\langle V\rangle$ with $k=2 \pi n / L$ and $\langle V\rangle=L^{-1} \int_{0}^{L} V d x$. The function $\sigma(x)$ is almost real. However, due to a weak disorder it acquires a small fluctuating imaginary part. Due to this 
imaginary part the logarithm of the modulus of the wave function (again in the leading order in $V$ ) behaves like

$$
\ln |\psi(x)|^{2}=\frac{h}{k^{2}+h^{2}} \int_{0}^{x}\left(V\left(x^{\prime}\right)-\langle V\rangle\right) d x^{\prime} .
$$

Thus we see that our wave function may be large (exponentially!) or small depending on the occasional sign of the integral. We see also that the momentum $k$ emerges in the Eq. (1) only in the overall factor $\left(k^{2}+h^{2}\right)^{-1}$. As a result all wave functions turn out to be localized at the same place. This feature of localization in the strong imaginary potential is in sharp contrast with what happens in the Hermitean $1 d$ disordered Hamiltonians, where eigenfunctions with close energy strongly repel each other in the coordinate space. In the case of white noise disorder $\overline{V(x) V\left(x^{\prime}\right)}=D \delta\left(x-x^{\prime}\right)$ one may find how fast the logarithm Eq. (何) changes from point to point. Namely

$$
\overline{(\ln |\psi(x)|-\ln |\psi(y)|)^{2}}=\frac{h^{2} D|x-y|(L-|x-y|)}{4\left(k^{2}+h^{2}\right)^{2} L} .
$$

In particular this equation allows us to estimate, how the wave function decreases after approaching the maximum. One may introduce via the Eq. (5) the typical size of the wave packet (analog of the localization length)

$$
\xi_{h} \sim \frac{\left(k^{2}+h^{2}\right)^{2}}{h^{2} D} .
$$

This localization length formally is of the same order of magnitude as the usual Anderson localization length in $1 d$ disordered wire $\xi_{A} \sim 1 / D$. However, the nature of this localization is completely different. In Anderson case all wave functions decrease at large distances like $\exp \left(-x / \xi_{A}\right)$. In our case the wave functions decrease like $\exp \left(-\right.$ const $\left.\sqrt{x / \xi_{h}}\right)$ and even the constant in the argument of the exponent fluctuates with $x$ and from sample to sample (no thermodynamic limit).

Our results Eqs. (3-6) are valid only far from the transition from real to complex spectrum in the limit of weak disorder. In order to study the more general situation, numerical simulations were performed. For numerical computations it is natural to consider the tight binding variant of the model (1)

$$
-t\left(e^{-h} \psi_{n+1}+e^{h} \psi_{n-1}\right)+V_{n} \psi_{n}=E \psi_{n} .
$$

The approximate solution of this equation may be easily found for the case $h \gg 1$ (compare also with 10])

$$
\begin{aligned}
& E_{k} \approx-t\left[e^{h-i \phi}+e^{-h+i \phi}\right] \approx-t e^{h-i \phi} ; \phi=2 \pi \frac{k}{N} \\
& \psi_{n}^{(k)}=\exp \left\{i n \phi+e^{-h+i \phi} \sum_{m=0}^{n-1}\left(V_{m}-\langle V\rangle\right)+O\left(e^{-2 h}\right)\right\} .
\end{aligned}
$$

Here integer $N$ and $k$ are the size of the closed chain and the number of solution (momentum), $\langle V\rangle=$
$N^{-1} \sum_{n=0}^{N} V_{n}$. For concrete calculation we have used $N=300, t=1, h=0.4$, and the random potential uniformly distributed within the range $-1.5<V_{n}<1.5$. Thus we consider the case of intermediate or even strong disorder. The eigenvalues on the complex plane for one realization of disorder are plotted on the insert on Fig. 1. The left segment of real eigenvalues includes 29 low energy states. The more interesting for us are the squared wave functions $\left|\psi_{n}^{(i)}\right|^{2}$ shown on the same figure (the wave functions are normalized to unity in the usual sense $\sum\left|\psi_{n}\right|^{2}=1$ and only the values $\left|\psi_{n}\right|^{2}>10^{-5}$ are shown). We have shown the few typical states well below the phase transition $i=9,10,11$ and the states around and above the transition $i=27,30,36,50$. The solutions are numbered in accordance with increase of ReE. First, one sees that all the states below and above the "mobility edge" are clearly localized. Second, this localization has a very different form below and above the transition. The consecutive real eigenfunctions $i=9,10,11$ practically do not overlap. Alternatively, the states above the transition (and even slightly below) turns out to be very similar. This features of the wave functions are in explicit accordance with what we expected from the analytic estimates for weak disorder. On the Fig. 2 we have shown the $\ln \left|\psi_{n}^{(i)}\right|^{2}$ for $i=4,50,150$. As we have mentioned before, up to exponentially small corrections the real wave functions may be obtained from the solutions with $h=0$ via $\psi_{n}(h)=e^{n h} \psi_{n}(h=0)$. In accordance with this prediction we see on the Fig. 3 for $i=4$ that the logarithm of the wave function decreases linearly from the maximum with different slopes to the left and to the right. Also as we expected, the logarithm of the complex functions resembles to large extent the Brownian path. Moreover, due to the Eq. (8) at the middle of the zone $(\phi=\pi / 4)$ the contribution of the leading order in $V$ changes only a phase of the wave function but not an amplitude. The Eq. (8) is valid only for $h \gg 1$ and this peculiarity at the center of the band should not necessary survive in our simulations. However, as we see, the effect of localization is strongly suppressed (but is not washed out completely!) for $i=150$.

In order to introduce the analog of orthonormal basis in non-Hermitean case one has to consider two sets of eigenvectors: $H \psi_{R}=E \psi_{R}$ and $\psi_{L}^{T} H=E \psi_{L}^{T}$. Up to now in fact we have considered only the $\psi_{R}$. For our specific choice of the Hamiltonian Eq. (11) evidently $\psi_{L}(E, h) \equiv \psi_{R}\left(E^{*},-h\right)$. Moreover, in the leading approximation, with only $\sigma_{0}$ left in the Eq. (2), one simply has $\psi_{L}(x) \psi_{R}(x)=$ const. Thus we see that the growth (exponential!) of $\psi_{R}$ at the maximum is compensated by the decrease of $\psi_{L}$. This compensation is not an artifact of approximation Eqs. (2,3). For example, following the authors of the Ref. [1], one may introduce the conserving current $J=\psi_{L}^{\prime} \psi_{R}-\psi_{L} \psi_{R}^{\prime}+2 h \psi_{L} \psi_{R} ; d J / d x=0$. This means that the strong effect of stochastic localiza- 
tion may be washed out if the observables are bilinear in $\psi_{R}$ and $\psi_{L}$, but should be seen if the observables depend on only one $\psi_{R}$ or $\psi_{L}$. Surprisingly one finds the examples of both kinds within the most popular application of non-Hermitean Quantum Mechanics. Namely, the authors of Refs. [1, 15] have used the Hamiltonian Eq. (11) for description of Abrikosov vortex depinning by transverse magnetic field in the hollow superconducting cylinder with random columnar defects. The probability to find vortex at the point $x$ on the transverse slice $\tau$ of the cylinder with length $L_{\tau}$ was shown to have the form $P(x, \tau) \equiv Z^{-1}\left\langle\Psi^{f}\left|e^{-\left(L_{\tau}-\tau\right) H}\right| x\right\rangle\left\langle x\left|e^{-\tau H}\right| \Psi^{i}\right\rangle$. For very large $L_{\tau}$ only the ground state contribution survives in the evolution operator $e^{-L_{\tau} H}$. In this case naturally [1, 16] $P(x, \tau) \sim \psi_{R}(x) \psi_{L}(x)$ for $\tau, L_{\tau}-\tau \sim L_{\tau}$. We see that the effect of localization above depinning transition, which we consider in this paper, could hardly be seen inside the cylinder [17]. However, at the top of cylinder $P\left(x, L_{\tau}\right) \sim \psi_{R}(x)$ and at the bottom $P(x, 0) \sim \psi_{L}(x)$ the "stochastic localization", which we have introduced, turns out to be of $100 \%$ importance. We leave the more detailed discussion of the localization above depinning in case of many vortices for a separate publication 18.

Up to now we have studied the effect of imaginary vector potential only in the $1 d$ systems. Consider now the closed strip of the width $l$ and length $L$ with random weak disorder $\overline{V(x, y) V\left(x^{\prime}, y^{\prime}\right)}=D_{2} \delta\left(x-x^{\prime}\right) \delta\left(y-y^{\prime}\right)$ and imaginary potential along the ring (the Hamiltonians of the kind Eq. (11) in more than $1 d$ have been also considered in Refs. 11,9, 11, 12). The Hamiltonian for the strip differs from that of the Eq. (11) by the only additional term $-\partial^{2} / \partial y^{2}$. It is convenient to look for the solutions of this Schrödinger equation of the form

$$
\psi(x, y)=\sum \psi_{n}(x) \sqrt{2 / l} \sin \left(q_{n} y\right),
$$

with $q_{n}=n \pi / l$. The analog of the Eq. (11) now reads

$$
-(\partial / \partial x-h)^{2} \psi_{n}+\sum_{m} V_{n m} \psi_{m}=\varepsilon_{n} \psi_{n},
$$

where the longitudinal energy $\varepsilon_{n}=E-q_{n}^{2}$. In the case of white noise disorder $\overline{V_{n n}(x) V_{n n}\left(x^{\prime}\right)}=\left(3 D_{2} / 2 l\right) \delta\left(x-x^{\prime}\right)$ and for $m$ and $n$ different $\overline{V_{n m}(x) V_{m n}\left(x^{\prime}\right)}=\left(D_{2} / l\right) \delta(x-$ $x^{\prime}$ ). At least for small $l$ (or large $\varepsilon_{n}-\varepsilon_{m}$ ) and for disorder weak enough one may consider the hopping $V_{n m}$ in Eq. (10) as a perturbation. The zeroth approximation for the wave function in this simple case is again $\psi_{n}^{(0)}=$ $\exp \left\{\int^{x}\left(\sqrt{\varepsilon_{n}-V_{n n}}-i h\right) d x^{\prime}\right\}$ with some given $n$ and $E=$ $\varepsilon_{n}+q_{n}^{2}=(k+i h)^{2}+q_{n}^{2}$. Physically interesting, however, is the range of validity of this single chanel approximation. The corrections to $\psi_{n}^{(0)}$ are described by the equation

$$
\left[-(\partial / \partial x-h)^{2}-\varepsilon_{m}\right] \psi_{m}^{(1)}=-V_{m n} \psi_{n}^{(0)} .
$$

The solution of this equation may be found by the Green's function method. Let for definiteness $\varepsilon_{m}-\varepsilon_{n}>0$ (note that $\operatorname{Im} \varepsilon_{m} \equiv \operatorname{Im} \varepsilon_{n}$ ). In this case the Green's function for the Eq. (11) $G(x-y)$ equals zero at $x>y$ and has a cusp at $x=y$. The solution reads

$$
\psi_{m}^{(1)}(x)=\int_{x}^{\infty} \frac{e^{\lambda_{1}(x-y)}-e^{\lambda_{2}(x-y)}}{\lambda_{2}-\lambda_{1}} V_{m n}(y) \psi_{n}^{(0)}(y) d y,
$$

where $\lambda_{1} \approx 2 h-i k$ and $\lambda_{2} \approx i k+\left(\varepsilon_{m}-\varepsilon_{n}\right) /(h-i k)$ ( $k$ is related with $E$ as we have shown above and we suppose that $\left.\left|\varepsilon_{m}-\varepsilon_{n}\right| \ll \varepsilon_{n}\right)$. For $|n-m| \ll n$ one has $R e \lambda_{2} \ll k, h$. However, if $\left(k^{2}+h^{2}\right)\left|\varepsilon_{m}-\varepsilon_{n}\right| \gg h D_{2} / l$ (compare with Eq. (6)) the amplitude of the exponent $\left|\exp \left(\lambda_{2} x\right)\right|$ still vary with $x$ much faster than $\left|\psi_{n}^{(0)}(x)\right|$. In this case the averaged value

$$
\overline{\left|\psi_{m}^{(1)}\right|^{2}}=\frac{D_{2}}{32 \pi h q} \frac{1}{n-m}\left|\psi_{n}^{(0)}(x)\right|^{2} .
$$

The analogous formula for $\varepsilon_{m}-\varepsilon_{n}<0$ is obtained after the replacement $n-m \rightarrow|n-m|$. In the similar way one may find the correction to the direct potential $V_{n n}$ induced by the hopping $V_{n m}$ in the second order of perturbation theory. This correction in its turn renormalizes the speed of stochastic growth of the amplitude of wave function Eq. (5) and the localization length Eq. (6). The generalization of the Eq. (5) for the strip gives

$$
\begin{aligned}
& \overline{\left(\ln \left|\psi_{n}^{(0)}(x) / \psi_{n}^{(0)}(y)\right|\right)^{2}}= \\
& \frac{h^{2}|x-y| D_{2}}{4\left(k^{2}+h^{2}\right) l}\left\{\frac{3}{2}+\frac{D_{2}}{32 \pi h q} \sum_{m \neq n} \frac{1}{|n-m|}+\ldots\right\} .
\end{aligned}
$$

Here $|x-y| \ll L$. The sum over $m$ in the r.h.s. is logarithmically divergent. The summation should be cut at $|n-m| \sim k l$ (we suppose that $k \sim q \sim h$ ). Thus we see that at small $l$ the growth of the wave function simply reproduces that of the single chanel case Eq. (5) with the effective localization length proportional to the width of the strip $\xi_{h} \sim l$. Only at $D_{2} \ln (k l) / h q \sim 1$ the features of wave functions in a strip became sufficiently different from those in 1-chanel wire. One may interpret this results as an indication of the existence of exponentially large localization length on $2 d$ plane with imaginary vector potential.

One may easily repeat the calculations Eqs. (9-14) also in the case of thick $3 d$ wire. The result of such generalization will be the formula analogous to the Eq. (14) and $\xi_{h} \sim l^{2} / D_{3}$ ( $l$ is the typical transverse size of the wire). Also like in $2 d$ case the new physics starts only at exponentially thick wire $D_{3} \ln (k l) / q \sim 1$. However, the concrete behaviour of the perturbed wave function may be completely different for $2 d$ and $3 d$. In a strip due to a complex energy $E=\left(k_{\|}+i h\right)^{2}+q_{\perp}^{2}$ there is no degeneracy of different transverse channels. Thus the only new phenomena we may expect with increase of the width of 
the strip is localization of wave functions in transverse direction due to the mixing of channels with very close $q_{\perp}$. In $3 d$ case the analog of the Eq. (13) describes the diffusive mixing of many channels with close $\left(\vec{q}_{\perp}\right)^{2}$ but completely different $\vec{q}_{\perp}$.

In summary, we have shown in this paper that the transition from real to complex spectrum in $1 d$ disordered systems with imaginary vector potential is not a delocalization transition. However, the nature of localization below and above the transition is completely different. The most interesting is the localization at complex energies, there the wave function decreases (increases) like the exponent of $\sqrt{x / \xi_{h}}$ and this exponent even has no a well defined thermodynamic limit. As for physical applications, the localization at complex energies becomes invisible for observable values bilinear in left- and right- eigenvectors of non-Hermitean Hamiltonian, but is of $100 \%$ importance for observables depending on only one of them $\psi_{L}$ or $\psi_{R}$. For example, the effect which we consider will lead to the strong modification of the distribution of flux lines at the ends of superconducting cylindrical shell with random columnar defects. For the $2 d(3 d)$ generalizations of the model the same effect takes place with the effective localization length $\xi_{h}$ proportional to the transverse size(area) at least until the system became exponentially thick.

Author is thankful to L. F. Khailo for help in computations. The work was supported by RFBR, grant 98-02-17905.

[1] N. Hatano and D. R. Nelson, Phys. Rev. Lett. 77, 570 (1996); ibid, Phys. Rev. B 56,8651 (1997)

[2] D. R. Nelson and N. M. Shnerb, cond-mat/9708071.

[3] K. B. Efetov, Phys. Rev. Lett. 79, 491 (1997); ibid, Phys. Rev. B 56,9630 (1997)

[4] J. Feinberg, A. Zee, cond-mat/9706218, cond-mat/9710040.

[5] P. W. Brouwer, P. G. Silvestrov, and C. W. J. Beenakker, Phys. Rev. B 56,R4333 (1997).

6] R. Janik, M. A. Nowak, G. Papp, and I. Zahed, condmat/9705098.

[7] I. Y. Goldsheid and B. A. Khoruzhenko, condmat/9707230.

[8] E. Brezin, A. Zee, cond-mat/9708029.

[9] C. Mudry, B. D. Simons and A. Altland, condmat/9712103.

[10] D. R. Nelson and N. M. Shnerb, cond-mat/9801111.

[11] A. Zee, cond-mat/9711114, to be published in Physica A.

[12] N. Hatano, cond-mat/9801283.

[13] The WKB approximation for investigation the spectrum of non-Hermitean Hamiltonians of the kind (1) was first used in Refs. [8,11.

[14] For fast fluctuating potential one should be careful with the high order terms of straightforward derivative expan- sion Eq. (8) ( $\sigma_{2}$ etc.). However, even for the white noise weak disorder all important features of the wave function are reproduced by the first term $\sigma_{0}$ in Eq. (2).

[15] D. R. Nelson and V. Vinokur, Phys. Rev. B 48,13060 (1993).

[16] U. C. Täuber and D. R. Nelson, Phys. Rep. 289, 157 (1997).

[17] Author is thankful to N. Hatano and A. Zee for discussion of this point.

[18] P. G. Silvestrov, in preparation.

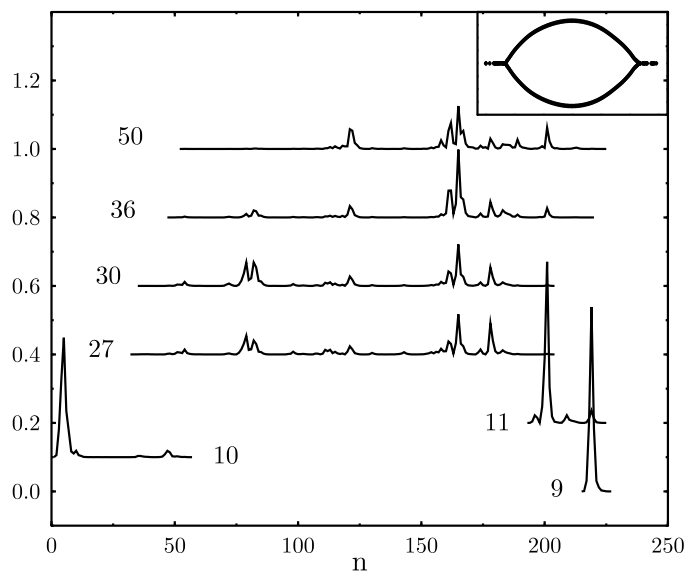

FIG. 1. The $\left|\psi_{n}^{(i)}\right|^{2}$ for the model Eq. (7) for states with numbers $i=9,10,11,27,30,36,50$. We use $N=300, t=1$, $h=0.4$, and $-1.5<V_{n}<1.5$. The first vector with complex energy is $i=30$. All states are clearly localized. However, the states far below the "mobility edge" differ strongly from each other, while the complex states look quite similar. Insert shows the eigenvalues on the complex plane.

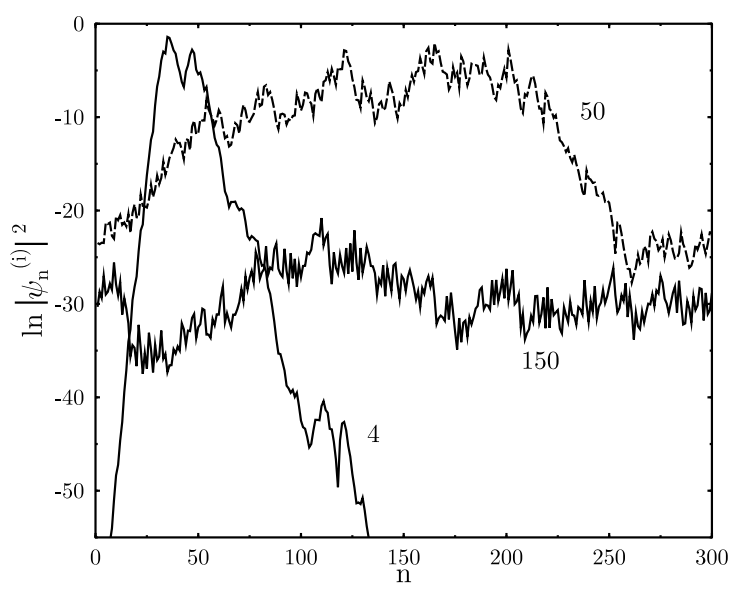

FIG. 2. The $\ln \left|\psi_{n}^{(i)}\right|^{2}$ for $i=4$ ("localized") and $i=50,150$ ("stochastic") states. The data for $i=150$ was multiplied by the factor 5. The Brownian curves for complex states and different left and right slope for $i=4$ are clearly seen. 